\title{
Supplemental nitric oxide and its effect on myocardial injury and function in patients undergoing cardiac surgery with extracorporeal circulation
}

Jacopo Gianetti, MD, PhD

Paolo Del Sarto, MD

Stefano Bevilacqua, MD

Cristina Vassalle, BSc

Rossella De Filippis, BSc

Mirsad Kacila, MD

Pier Andrea Farneti, MD

Aldo Clerico, MD

Mattia Glauber, MD

Andrea Biagini, MD

From the CNR Institute of Clinical Physiology, G. Pasquinucci Hospital, Massa, Italy.

Received for publication March 26, 2002; revisions requested May 16, 2002; revisions received June 3, 2002; accepted for publication Aug 16, 2002.

Address for reprints: Jacopo Gianetti, MD, $\mathrm{PhD}$, G. Pasquinucci Hospital, CNR, Institute of Clinical Physiology, Via Aurelia Sud, 54100 Massa, Italy (E-mail: gianetti@ifc.cnr.it).

J Thorac Cardiovasc Surg 2004;127:44-50 $0022-5223 / \$ 30.00$

Copyright $\odot 2004$ by The American Association for Thoracic Surgery

doi:10.1016/j.jtcvs.2002.08.001
Background: Cardiopulmonary bypass induces a systemic inflammatory response that may contribute to clinical morbidity. Gaseous nitric oxide at relatively low concentrations may elicit peripheral anti-inflammatory effects in addition to a reduction of pulmonary resistances. We examined the effects of $20 \mathrm{ppm}$ of inhaled nitric oxide administered for 8 hours during and after cardiopulmonary bypass.

Methods and Results: Twenty-nine consecutive patients undergoing aortic valve replacement combined with aortocoronary bypass were randomly allocated to either $20 \mathrm{ppm}$ of inhaled nitric oxide $(\mathrm{n}=14)$ or no additional inhalatory treatment $(\mathrm{n}=$ 15). Blood samples for total creatine kinase, creatine kinase MB fraction, and troponin I measurements were collected at $4,12,24$, and 48 hours postsurgery. In addition, we collected perioperative blood samples for measurements of circulating nitric oxide by-products and brain natriuretic peptide. Soluble P-selectin was analyzed in blood samples withdrawn from the coronary sinus before and after aortic clamping. The area under the curve of creatine kinase MB fraction $(P=.03)$, total creatine kinase $(P=.04)$, and troponin I $(P=.04)$ levels were significantly decreased in the nitric oxide-treated patients. Moreover, in the same group we observed blunted P-selectin and brain natriuretic peptide release $(P=.01$ and $P=$ .02 , respectively). Nitric oxide inhalation consistently enhanced nitric oxide metabolite levels $(P=.01)$.

Conclusions: Nitric oxide, when administered as a gas at low concentration, is able to blunt the release of markers of myocardial injury and to antagonize the left ventricular subclinical dysfunction during and immediately after cardiopulmonary bypass. The organ protection could be mediated, at least in part, by its antiinflammatory properties.

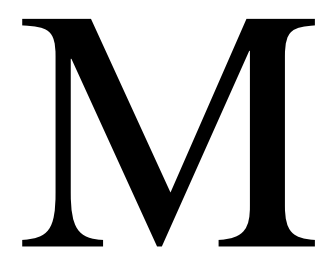

any factors during cardiopulmonary bypass (CPB), both material-dependent and material-independent, have been documented to induce a complex inflammatory response. ${ }^{1}$ This inflammatory cascade may contribute to the development of postoperative complications, including respiratory failure, renal dysfunction, bleeding disorders, neurologic dysfunction, altered liver function, myocardial injury, and, finally, multiple organ failure. 
Oxygen-derived free radicals are primarily involved in ischemia-reperfusion injury, maximally affecting the myocardium at the onset of reperfusion, after release of aortic clamp. ${ }^{2}$ Reperfusion injury has a complex pathophysiology. It develops over a period of several (2-24) hours and involves 2 distinct but related events, coined "the endothelial trigger" and "the neutrophil amplification" step. ${ }^{3}$ One of the major early events leading to endothelial dysfunction is the loss of the release of nitric oxide (NO) by endothelial cells, which occurs within a few minutes of reestablishing flow. This finding has been demonstrated in both an intact animal subjected to myocardial ischemia-reperfusion and in an isolated perfused organ (the heart) subjected to reperfusion with a blood-free solution. ${ }^{4}$ The endothelial dysfunction is followed by a sequence of events that results from the altered status of the endothelium. The second major step in the process of reperfusion injury is the "neutrophil amplification step," which involves leukocyte-endothelial cell interactions leading to leukocyte adherence to the endothelium and transendothelial migration of neutrophils across the endothelium. ${ }^{5}$ There is a dramatic increase in neutrophil adherence, which reaches its maximum at 20 minutes postreperfusion. ${ }^{6}$ These data strongly implicate the role of adhesion molecule expression and especially of P-selectin, which are up-regulated within a few minutes. ${ }^{7}$ This enhanced leukocyte adherence to the endothelium leads to capillary plugging and edema formation, resulting in reduction of coronary blood flow. The response to ischemiareperfusion injury is termed the "no-reflow phenomenon." These events culminate in a profound degree of myocardial cell necrosis and/or apoptosis at 4 to 24 hours postreperfusion, with early and late reduction of myocardial contractility. ${ }^{9}$

Because reduced NO release is proven to be a marked and early event in the reperfusion injury, we speculated that NO replacement therapy could blunt the consequences of ischemia-reperfusion injury. One component of the reduced NO release is the enhanced quenching of NO by superoxide radicals, which appears to be related to superoxide radicals produced by the abrupt reoxygenation of the reperfusion process. ${ }^{10}$

The present investigation addresses the beneficial effect of inhaled NO, administered at pharmacological dosages (20 ppm) during and for 8 hours after CPB, in minimizing the release of markers of myocardial injury and left ventricular dysfunction in patients undergoing CPB.

\section{Methods \\ Study Design}

This investigation was designed as a prospective, randomized, nonblinded study carried out at the Pasquinucci Hospital in Massa, Italy, between July 2000 and April 2002. After approval of the Local Ethical Commission, informed consent was obtained from 29 patients undergoing nonemergency aortic valve replacement
TABLE 1. Demographic, clinical, and surgical characteristics

\begin{tabular}{lcc}
\hline Parameter & $\begin{array}{c}\text { N0 group } \\
\text { (n = 14) }\end{array}$ & $\begin{array}{c}\text { Control group } \\
\text { (n= 15) }\end{array}$ \\
\hline Age (years) & $70 \pm 13$ & $69 \pm 10$ \\
Gender (number of men) & 9 & 8 \\
Current smokers & 5 & 5 \\
Arterial hypertension & 8 & 9 \\
Creatininemia (mg/dL) & $1.2 \pm 0.5$ & $1.1 \pm 0.7$ \\
Triglycerides (mg/dL) & $128 \pm 41$ & $133 \pm 53$ \\
LDL cholesterol (mg/dL) & $106 \pm 40$ & $102 \pm 35$ \\
Type-2 diabetes & 3 & 4 \\
Number of coronary artery disease & $2 \pm 1.0$ & $2 \pm 0.9$ \\
Parsonnet index (23) & $3 \pm 1.2$ & $2.6 \pm 0.8$ \\
Preoperative ejection fraction (\%) & $57 \pm 9$ & $54 \pm 9$ \\
Time of extracorporeal circulation & $107 \pm 40$ & $110 \pm 35$ \\
$\quad$ (min) & & \\
Time of aortic clampage (min) & $76 \pm 12$ & $78 \pm 16$ \\
Time of intubation (h) & $14 \pm 6$ & $14 \pm 5$ \\
Time in intensive care unit (h) & $35 \pm 21$ & $35 \pm 16$ \\
\hline
\end{tabular}

$L D L$, Low-density lipoprotein; $N O$, nitric oxide.

combined with aortocoronary bypass, and the patients were enrolled in the present study.

Patients with an active infection, ejection fraction $<30 \%$, malignancy, or a history of hematologic, hepatic, or renal disorders were excluded. Exclusion criteria also included corticosteroid or a nonsteroidal anti-inflammatory treatment within the previous 7 days or during surgery, or a postoperative treatment with nitrates or sodium nitroprussiate.

\section{Study Procedures}

All potential patients were screened before enrollment. When all inclusion and exclusion criteria were satisfied, patients were randomized. Perioperative testing included medical history and physical examination, laboratory testing (hematology, chemistry, and urinalysis), electrocardiogram, and echocardiography. Demographic characteristics and surgical data are reported in Table 1.

\section{Surgical Protocol}

The surgical protocol consisted of conventional median sternotomy access and the institution of CPB using a double-stage venous cannula in the right atrium and an arterial cannula in the ascending aorta. All procedures were performed in mild hypothermia (32$35^{\circ} \mathrm{C}$ ), using retrograde, intermittent, hematic hyperkalemic cardioplegic solution through the coronary sinus.

\section{NO Delivery, Systems, and Timing}

NO gas was mixed with nitrogen (500 ppm). The mixture of gases was continuously introduced into the patient's ventilatory circuit and was connected to a time-cycled anesthesia machine ventilator (Servo 900D; Siemens-Elema, Stockholm, Sweden). The NO gas inlet was proximally connected to the inspiratory part of the patient ventilatory circuit. The concentration of NO delivered was sidestream analyzed by a chemiluminescent instrument (Nox Box Bedfont Scientific, Kent, United Kingdom) connected to the distal inspiratory limb of the ventilatory circuit. The exhaled gas and 


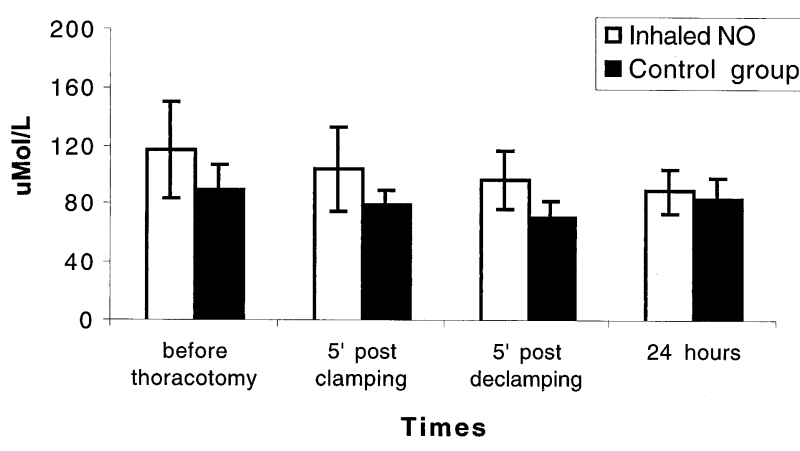

Figure 1. Results of colorimetric assay for $\mathrm{NO}$ by-products (NO ${ }^{-2}$ and $\mathrm{NO}^{-3}$ ) on blood samples peripherally drawn at prefixed perioperative intervals: $T 1$, prethoracotomy; $T 2,5$ minutes after aortic clamping; T3, 5 minutes after aortic unclamping; T4, 24 hours after surgery. Data shown are mean \pm SEM for patients who received NO (lighter blocks) and for controls (darker blocks). ${ }^{*} P<.05$.

gases exiting the chemiluminescence instrument were collected into a Venturi device (Servo 900D; Siemens-Elema, Stockholm, Sweden) and discharged into the hospital vacuum system. At the onset of CPB (when 100\% oxygen is usually delivered to the membrane oxygenator), NO was delivered to the oxygenator with our hospital's standardized oxygen/air mixture flow into the side port of the inlet gas tubing, close to the flowmeters. The cardioplegic solution circuit was designed to administer oxygenated hyperkalemic blood enriched with NO. The NO analyzing instrument was connected at the distal part of the gas tubing, close to the oxygenator. The delivery of NO was adjusted using a flowmeter on the cylinder manifold to provide a constant preset concentration of NO. The fine-tuning of the NO concentration delivered was corrected on the basis of the chemiluminescent analysis. The NO was administered to each patient during the entire period of intubation, from the operating theater to the intensive care unit. In particular, NO delivery was started immediately after patient intubation in the operating theater via the ventilatory circuit, then continued throughout the CPB directly into the pump prime and in the intensive care unit via the ventilator circuits until suspension of mechanical ventilation. The gas outflow from the membrane oxygenator was scavenged using the wall-mounted vacuum system. During inhalation, hemodynamic variables were monitored by routine cardiac catheterization. A standard blood gas analyzer was used to measure $\mathrm{PaO}_{2}, \mathrm{PaCO}_{2}$, and $\mathrm{pH}$.

\section{Blood Sample Collection}

Blood samples for indices of myocardial injury were drawn before surgery and $4,12,24$, and 48 hours after surgery. NO by-products were measured in blood samples taken prethoracotomy (before heparinization), 5 minutes post-aortic clamping, 5 minutes postaortic unclamping, and 24 hours postsurgery, approximately 12 hours after NO was stopped. Analogously, for brain natriuretic peptide (BNP) measurements we used peripheral blood samples drawn prethoracotomy (before heparinization), 5 minutes postaortic clamping, 5 minutes post-aortic unclamping, 5 minutes post-CPB, and 4 and 24 hours postsurgery. Blood samples from coronary sinus were taken before and after cardioplegia for Pselectin measurements. All samples were immediately centrifuged at $4^{\circ} \mathrm{C}$ and stored at $-80^{\circ} \mathrm{C}$ until they were assayed.

\section{Biological Assays}

Soluble P-selectin was measured by a double sandwich enzymelinked immunosorbent assay (Bender, Vienna, Austria) in heparinized plasma samples. ${ }^{11}$ Assays were performed in duplicate on duplicate samples, each assayed at least at 2 different dilutions. Values obtained in healthy people ranged between 100 and 300 $\mathrm{ng} / \mathrm{mL}$, in accordance with the literature. ${ }^{11}$ Plasma BNP was measured by a noncompetitive immunofluorometric assay (Triage BNP Test, Biosite Diagnostics, Inc, San Diego, Calif), as described elsewhere. ${ }^{12}$ The imprecision of the Triage method was about $12 \%$ for BNP concentrations in the normal range and about $18 \%$ for BNP concentrations above the normal range. The working range was 5 to $1300 \mathrm{pg} / \mathrm{mL}$.

\section{Measurements of NO Metabolites}

To analyze the NO metabolites (NOx), the plasma samples were ultrafiltered through $30-\mathrm{KDa}$ molecular weight cutoff filters and centrifuged at $1000 \mathrm{~g}$ for 60 minutes to remove hemoglobin, which is known to interfere with subsequent spectrophotometric measurements. Afterward, NOx concentration was determined by a colorimetric assay kit (Cayman, Ann Arbor, Mich) based on Griess reaction. ${ }^{13}$ Standards for calibration curves were prepared with nitrate and taken through the full assay procedure. The results were expressed as $\mu \mathrm{mol} / \mathrm{L}$ of NOx.

\section{Creatine Kinase-MB and Troponin I Assay}

Myocardial-specific isoforms of creatine kinase (CK) and troponin I were measured in our clinical laboratory by a point-of-care immunometric technique (Triage cardiac panel kit).

\section{Statistical Analysis}

All data are presented as mean \pm standard error of the mean (SEM). Statistical analysis was performed by multivariate analysis of variance for repeated measurements for a comparison among more than 2 groups and Student $t$ test for a comparison between 2 groups; correlation among variables was estimated by a linear regression analysis with the aid of the Statview statistical package (Abacus Concepts, Inc, Berkeley, Calif).

\section{Results}

\section{Population Characteristics}

Demographic variables, chemistry laboratory values, and surgical parameters (Table 1) did not significantly differ between the 2 patient groups.

\section{NO By-product Measurements}

Measurements of NO metabolites showed significant intraand intergroup differences: in the NO group the peak level was observed at time T1, corresponding to the intraoperatory sample, before thoracotomy, 10 minutes after starting NO inhalation (Figure 1). NO metabolites continued to exhibit significantly higher levels compared with basal val- 
ues $(P=.02)$ at 5 minutes post-aortic clamping (T2) and 5 minutes post-aortic unclamping (T3), corresponding to the extracorporeal circulation period, when NO was administered in the membrane oxygenator as described. At time T4, 24 hours after surgery and about 12 hours after stopping NO inhalation, NO by-products completely reverted to basal levels. In the control group we did not observe a significant increase in NO by-products during the extracorporeal circulation period compared with basal values. Overall comparison between NO and control groups indicated a statistically significant difference $(P=.01)$.

\section{Analysis of Myocardial Injury}

Indices of myocardial injury showed a constant increase throughout the first 24 hours after surgery (Figure 2). Comparison between groups for total CK release indicated a statistically significant difference at 24 and 48 hours $(P=$ .02 ; Figure $2, A$ ) and for the cumulative release summarized by the area under the curve (AUC; $P=.04$; Figure 2, $B$ ). Analogously, we found the peak levels of creatine kinase MB fraction $(\mathrm{CK}-\mathrm{MB})$ at 24 hours $(P=.01)$; values at 48 hours $(P=.01$; Figure $2, C)$ and the AUC $(P=.03$; Figure $2, D)$ were significantly lower in the NO group as compared with the control group. Moreover, in the same group the AUC of troponin I was lower than in the control group $(P=$ .04 ; Figure 3).

\section{Analysis of Endothelial Cell Activation}

In both groups we observed a relevant increase of soluble $\mathrm{P}$-selectin in plasma samples collected from coronary sinus immediately before and after aortic clamping (Figure 4, A). The amount of this inflammatory release, which was also calculated as a pre-/post-aortic clampage gradient (Figure $4, B)$, turned out to be significantly blunted in the NO group as compared with the control group $(P=.02)$.

\section{Analysis of Left Ventricular Dysfunction}

To normalize the basal values (T1), we analyzed the gradient of BNP release as measured by the percent of the ratio between the levels at each time (T2-T6) compared with T1 (Figure 5). We observed a statistically significant difference at T5 $(P=.02)$, with lower levels $(249 \pm 71$ vs $311 \pm 141$ $\mathrm{pg} / \mathrm{mL}$ ) in the NO group.

\section{Discussion}

Effective inhibition of CPB-induced inflammatory reaction and of ischemia-reperfusion injury following coronary artery bypass surgery has the potential to significantly reduce clinical morbidity. ${ }^{14}$ These phenomena are both present in patients undergoing aortic valve replacement and coronary artery bypass revascularization.

We report that administration of NO via inhalation immediately before, during, and after surgery may positively

\section{Peri-operative time-course: total CK release}

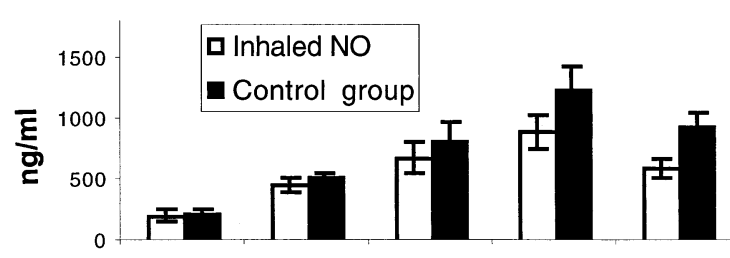

A

Times

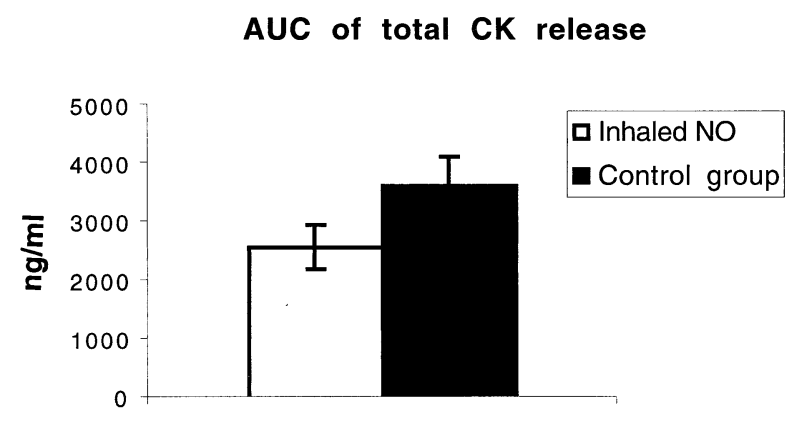

B

Peri-operative time-course: CK-MB release

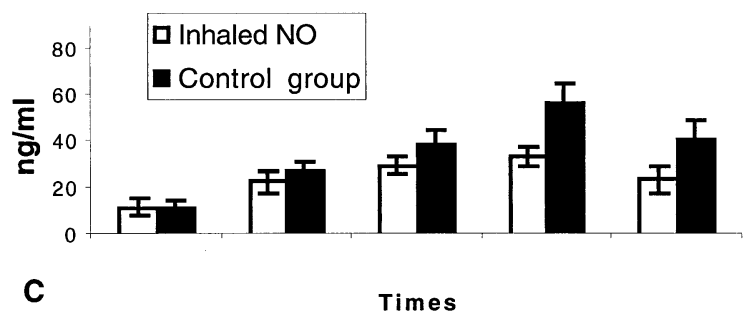

AUC of CK-MB release

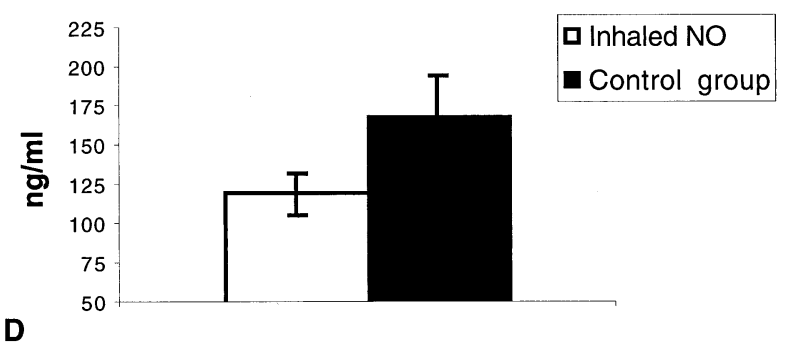

Figure 2. Analysis of myocardial injury determined in CPB patients by measurements of the release of total CK (A) and CK-MB (C) and by measurements of the cumulative release (AUC) of total CK (B) and CK-MB (D) over 48 hours postsurgery. Data shown are mean \pm SEM for patients who received NO (lighter blocks) and for controls (darker blocks). ${ }^{*} P<.05$. 


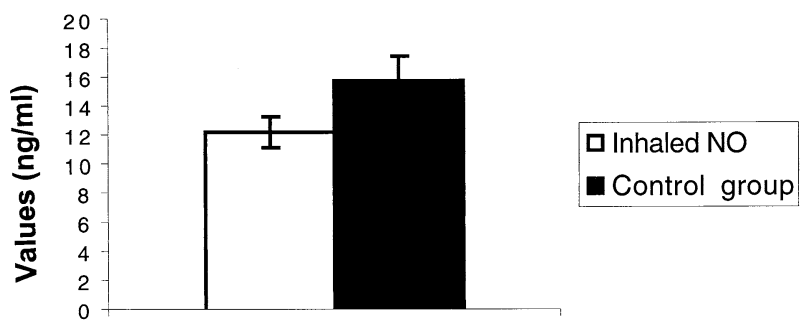

Figure 3. Analysis of myocardial injury determined in CPB patients by measurements of the release of total troponin I over 48 hours postsurgery. Data shown are mean \pm SEM for patients who received NO (lighter blocks) and for controls (darker blocks). *P $<.05$.
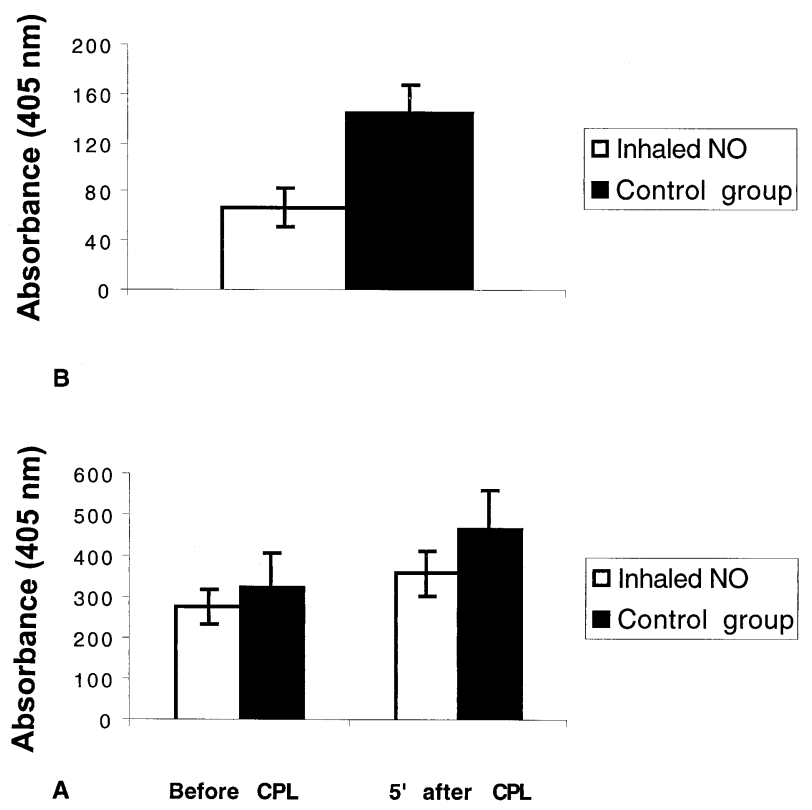

Figure 4. Analysis of early endothelial activation in CPB patients by measurements of soluble P-selectin in blood samples taken from coronary sinus before and 5 minutes after the end of aortic clamping (A). Gradient of soluble P-selectin between pre-/postaortic clamping in both patients groups are illustrated (B). Data shown are mean \pm SEM for P-selectin measurements for patients who received NO (lighter blocks) and for controls (darker blocks). ${ }^{*} \boldsymbol{P}<.05$.

affect the pattern of release of markers of myocardial injury and left ventricular dysfunction, independently on its hemodynamic effects on the pulmonary vasculature and arterial saturation.

A major pathophysiological clue of the post-CPB reaction is an overall decrease of the endothelial NO synthase, responsible for a deficient vasodilatory tendency of the microvasculature and for an increased permeability of the endothelium toward circulating leukocytes. ${ }^{15}$ As a consequence, an inflammatory infiltration spreads out inside pe-

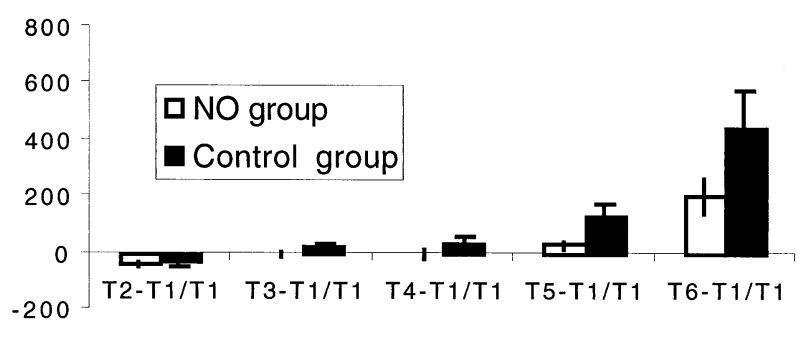

\section{Conditions}

Figure 5. BNP release in blood samples peripherally drawn at prefixed perioperative intervals: T1, prethoracotomy; T2, 5 minutes after aortic clamping; T3, 5 minutes after aortic unclamping; T4, 5 minutes after extracorporeal circulation; T5, 6 hours after surgery; T6, 24 hours after surgery. To normalize the basal values, we report the gradient of BNP release as measured by the percent of the ratio between the levels at each time (T2-T6) compared with T1. Data shown are mean \pm SEM for patients who received NO (lighter blocks) and for controls (darker blocks). *P $<.05$.

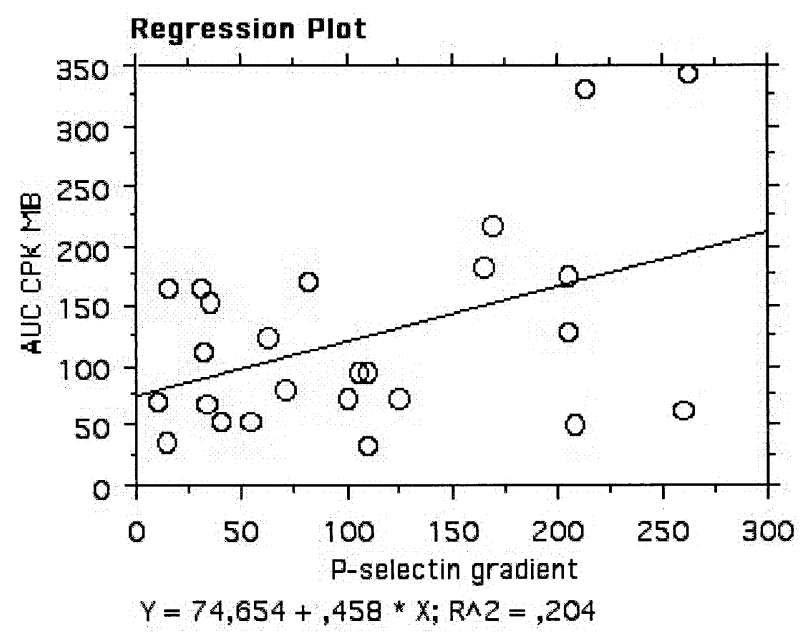

Figure 6. Simple regression analysis relating the extent of myocardial injury as detected by the AUC of CK-MB to the gradient of soluble P-selectin before/after aortic clamping.

ripheral tissues, releasing toxic mediators that further amplify the inflammatory reaction and cause organ injury. ${ }^{16}$ In this context, restoring NO production is theoretically beneficial. NO inhalation is an alternative route of administration of NO, with peculiarities that can account for specific advantages versus other approaches. NO easily diffuses through the air-blood barrier into the pulmonary circulation. NO inhalation in a low dosage causes selective pulmonary vasodilation. ${ }^{17}$ In this district NO diffuses into the smooth muscle cells, increasing cyclic guanylate monophosphate (cGMP) concentrations, whereas it does not cause systemic hypotension, being quickly inactivated by avid binding to hemoglobin. ${ }^{18}$ In addition to these proper- 
ties, inhaled NO can mediate some anti-inflammatory effects directly on the circulating leukocytes and platelets. In fact, NO gas inhibits human platelet aggregation, P-selectin expression, and fibrinogen binding in vitro and in vivo. ${ }^{19}$ If the concentration of inhaled NO in the inspiratory gas is below $80 \mathrm{ppm}$ we do not expect pro-oxidant effects. ${ }^{20}$ Interestingly, $\mathrm{NO}$ donors have variable rates of $\mathrm{NO}$ release and some of these substances release different molecules, with potential collateral effects. ${ }^{21}$

By measuring NO by-products in peripheral plasma samples we were able to document a significant increase during NO therapy beginning with the ventilatory phase and continuing throughout the extracorporeal circulation (T2 and T3), although at a lower rate. As we measured peak levels at time $\mathrm{T} 1$, just 10 to 15 minutes after starting inhalation, we confirmed the fast action of inhaled NO provided by this route of administration. Lower levels at T2 and T3 as compared with $\mathrm{T} 1$ indicated a less efficient diffusion of $\mathrm{NO}$ through the membrane oxygenator as compared with its diffusion across the alveolar-blood barrier. Whether the NO blood concentrations during this phase were adequate or not is a matter needing further investigation; neither is there a clear-cut idea about the possible correlation between NO by-products levels and clinical outcome.

At $20 \mathrm{ppm}$ of inhaled NO we failed to observe any effects on peripheral vascular resistance, whereas the pulmonary vascular resistance was significantly decreased, as expected (75 \pm 8 vs $125 \pm 16$ dynes $\left.\cdot \mathrm{s} / \mathrm{cm}^{-5}, P=.002\right)$. Moreover, NO and control groups showed comparable results in term of arterial oxygen saturation and arterial tension of oxygen, time of intubation, time in intensive care unit, and postoperative ejection fraction (data not shown). Even in a relatively small number of patients we report a significant protection against perioperative release of markers of myocardial injury, as indicated by the overall reduced amounts of total CK, CK-MB, and troponin I levels throughout the first 24 hours after surgery. This effect was more impressive in patients with a lower release of soluble P-selectin in the coronary sinus, measured by the pre- $/$ postcardioplegia gradient, as shown by the correlation between the total amount of CK-MB release and the soluble P-selectin gradient in the local circulation (Figure 6).

This observation, even if it cannot be interpreted as a definitive explanation of the NO biological action, may suggest the hypothesis that $\mathrm{NO}$ supplementation in the local circulation, by providing NO directly in the cardioplegic solution, has a significant anti-inflammatory effect, per se, cardioprotective.

This finding is just a preliminary report suggesting a potential therapeutic effect of supplemental NO administered before, during, and after CPB. Already some evidence of the beneficial properties of inhaled NO on ischemiareperfusion injury are available in animal models. In 1996 in a canine model of platelet-mediated reocclusion after thrombolysis, inhaled NO improved the coronary artery patency rate. ${ }^{22}$

Moreover, the observation in the NO group of lower levels of BNP release, a surrogate of early left ventricular dysfunction as recently suggested by the European Task Force for the Diagnosis and Treatment of Heart Failure ${ }^{24}$ (Figure 5), supports the hypothesis of a real cardioprotective effect of the NO supplementation. Because the cardioplegic circuit was designed to administer oxygenated hyperkalemic blood enriched with NO, thus delivering the same amount of $\mathrm{NO}$ as the peripheral blood circulating in the extracorporeal circuit, this method of administration may be the best access route of therapeutic NO. In fact, whereas the pulmonary inhaled NO is almost completely metabolized before reaching the left atrium, NO supplemented in the cardioplegic solution directly diffuses in the coronary circulation and may elicit a local therapeutic effect.

\section{Limitations of the Study}

Several points regarding the effect of supplemented NO are lacking and need further investigation, such as the optimal duration of NO treatment, a dose titration of inhaled NO, a time course of $\mathrm{NO}$ by-products to establish how long-lasting the effect is, and how soon after stopping NO inhalation the rate of NO by-products falls.

Moreover, the inclusion of patients with combined coronary artery disease could actually add a confounding variable in our analysis; however, major surgical parameters in the 2 randomized groups were equivalent, including coronary artery disease, the time of $\mathrm{CPB}$, and the time of aortic clamping (Table 1). Finally, our study had a short followup, limited to the in-hospital period, and was lacking of major clinical end points that could support the cardioprotective effect of NO. This work is just a pilot and observational study, based on data of biochemical markers of myocardial injury and left ventricular dysfunction, and requires confirmation by a larger interventional study to verify the opportunity to extend the use of NO in clinical settings involving ischemia-reperfusion damage.

We thank the Cardiac Surgical Nursing Staff at the Pasquinucci's Hospital for their help in blood sample collection and the local biological staff for their help in blood sample storage.

\section{References}

1. Wan S, LeClerc JL, Vincent JL. Inflammatory response to cardiopulmonary bypass. Chest. 1997;112:676-92.

2. McCord JM. Oxygen derived free radicals in post-ischemic tissue injury. N Engl J Med. 1985;312:159-63.

3. Engler RL, Dahlgren MD, Morris DD, Paterson MA, Schmidt-Schonbein AW. Role of leukocytes in response to acute myocardial ischemia-reflow in dogs. Am J Physiol. 1986;251:H314-23.

4. Tsao PS, Aoki N, Lefer DJ, Johnson G, Lefer AM. Time course of endothelial dysfunction and myocardial injury during myocardial ischemia and reperfusion in the cat. Circulation. 1990;82:1402-12. 
5. Smith CW, Entman ML, Lane CL, et al. Adherence of neutrophils to canine cardiac myocites in vitro is dependent on intercellular adhesion molecule-1. J Clin Invest. 1991;88:1216-23.

6. Lefer AM, Tsao PS, Lefer DJ, Ma XL. Role of endothelial dysfunction in the pathogenesis of reperfusion injury following myocardial ischemia. FASEB J. 1991;5:2029-34.

7. McEver RP. GMP-140: a receptor for neutrophils and monocytes on activated platelet and endothelium. J Cell Biochem. 1991;45:156-61.

8. Engler RL, Schmidt-Schonbein GW, Pavlec RS. Leukocyte capillary plugging in myocardial ischemia and reperfusion in the dog. Am J Pathol. 1983;111:98-111.

9. Lefer A, Lefer DJ. The role of nitric oxide and cell adhesion molecules on the microcirculation in ischaemia-reperfusion. Cardiovasc Res. 1996;32:743-51

10. Ma XL, Weyrich AS, Lefer DJ, Lefer AM. Diminished basal NO release after myocardial ischaemia and reperfusion promotes neutrophils adherence to coronary endothelium. Circ Res. 1993;72:403-12.

11. Mulvihill NT, Foley JB, Murphy R, Crean P, Walsh M. Evidence of prolonged inflammation in unstable angina and non-Q wave myocardial infarction. J Am Coll Cardiol. 2000;36:1210-16.

12. Del Ry S, Giannessi D, Clerico A. Plasma brain natriuretic peptide measured by fully automated immunoassay and by immunoradiometric assay compared. Clin Chem Lab Med. 2001;39:446-50.

13. Green LC, Wagner DA, Glogowski J, Skipper PL, Wishnok JS, Tannenbaum ST. Analysis of nitrate, nitrite and $\left({ }^{15} \mathrm{~N}\right)$ nitrate in biological fluids. Anal Biochem. 1982;126:131-8.

14. Fitch JCK, Rollins S, Matis L, et al. Pharmacology and biological efficacy of a recombinant, humanized, single chain antibody $\mathrm{C} 5 \mathrm{com}-$ plement inhibitor in patients undergoing CABG with CPB. Circulation. 1999;100:2499-506.
15. Elgebaly SA, Houser SL, El Kerm AF, et al. Evidence of cardiac inflammation after open heart operations. Ann Thorac Surg. 1994;57: 391-6.

16. Wan S, LeClerc JL, Vincent JL. Inflammatory response to cardiopulmonary bypass. Chest. 1997;112:676-92.

17. Frostell C, Fratacci MD, Wain JC, Jones R, Zapol WM. Inhaled nitric oxide. A selective pulmonary vasodilator reversing hypoxic pulmonary vasoconstriction. Circulation. 1991;84:2212-16.

18. Rimar S, Gillis CN. Selective pulmonary vasodilation by inhaled nitric oxide is due to hemoglobin inactivation. Circulation. 1993; 88:2884-7.

19. Gries A, Bode C, Peter K, et al. Inhaled nitric oxide inhibits human platelet aggregation, P-selectin expression, and fibrinogen binding in vitro and in vivo. Circulation. 1998;97:1481-7.

20. Cuthbertson BH, Dellinger P, Dyar OJ, et al. UK guidelines for the use of inhaled nitric oxide therapy in adult ICUs. American-European Consensus Conference on ALI/ARDS. Intensive Care Med. 1997;23: 1212-18.

21. Aleryani S, Milo E, Kostka P. Formation of peroxynitrite during thiol-mediated reduction of SNP. Biochim Biophys Acta. 1999;1472: 181-90.

22. Adrie $\mathrm{C}, \mathrm{Bloch} \mathrm{K}$, Moreno $\mathrm{P}$, et al. Inhaled $\mathrm{NO}$ increases coronary artery patency after thrombolysis. Circulation. 1996;94:1919-26.

23. Bernstein AD, Parsonnet V. Bedside estimation of risk as an aid for decision-making in cardiac surgery. Ann Thorac Surg. 2000;69: 823-8.

24. Task Force, European Society of Cardiology. Guidelines for the diagnosis and treatment of chronic heart failure. Eur Heart J. 2001;22:1527-60.

\section{JTCVS On-Line Manuscript Submission and Review Please visit http://www.editorialmanager.com/jtcvs/}

Effective September 15, 2001, authors and reviewers may submit manuscripts and reviews electronically via Editorial Manager, our new Web-based system with full electronic submission, review, and status update capabilities.

As we move from paper to electronic submissions, the Editorial Office will make proxy submissions of all manuscripts accompanied by a diskette containing the electronic files of the text, tables, and figures. Editors, authors, and reviewers will receive automatic e-mails when significant events occur.

We strongly encourage all authors and reviewers to use Editorial Manager. Although we will continue to accommodate the submission of paper manuscripts for some months, our goal is to be completely electronic within 9 to 12 months.

All individuals currently in our database for whom we have e-mail addresses will receive via e-mail a system-assigned username and password that can be used to log in to the system without prior registration. All those not receiving the e-mail must register the first time they use the system.

As with any broad systemic change, the conversion to the new system will take some time to complete. We ask your patience as we replace our in-office database with the new system. We also encourage you to take advantage of the speed and efficiency that the new system will provide for us all: editor, author, reviewer, and publisher. 